Jansma, J.D., Wagner, C., Bijnen, A.B. A patient safety curriculum for medical residents based on the perspectives of residents and supervisors. Journal of Patient Safety: 2011, 7(2), 99-105

\begin{tabular}{|c|c|}
\hline Postprint Version & 1.0 \\
\hline Journal website & $\begin{array}{l}\text { http://journals.lww.com/journalpatientsafety/pages/articleviewer.aspx?year=201 } \\
\text { 1\&issue=06000\&article=00008\&type=abstract }\end{array}$ \\
\hline Pubmed link & http://www.ncbi.nlm.nih.gov/pubmed/21577078 \\
\hline DOI & 10.1097/PTS.0b013e31821b3ace \\
\hline
\end{tabular}

This is a NIVEL certified Post Print, more info at http://www.nivel.eu

\title{
A Patient Safety Curriculum for Medical Residents Based on the Perspectives of Residents and Supervisors
}

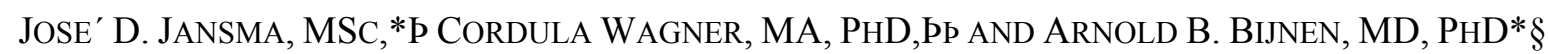

From the *Foreest Medical School, Medical Center Alkmaar, Alkmaar; †EMGO Institute for Health and Care Research, VU University Medical Center; $¥$ NIVEL Netherlands Institute for Health Services Research, Utrecht; and §VU University Medical Center, Institute for Education and Training, Amsterdam, The Netherlands.

Correspondence: Jose' D. Jansma, MSc, Foreest Medical School Medical Center Alkmaar, Wilhelminalaan 12, Room 054, 1815JD Alkmaar, The Netherlands (e-mail: j.d.jansma@mca.nl).

With financial support from Foreest Medical School of Medical Center Alkmaar, and the Ministry of Health, Welfare and Sport in the Netherlands, this study was conducted as part of the Dutch Patient Safety Research Program. This Program is initiated by the Dutch Society of Medical Specialists (in Dutch: Orde van Medisch Specialisten) and the Dutch Institute for Healthcare Improvement (CBO) and is carried out by EMGO Institute for Health and Care Research, VU University Medical Center/NIVEL Netherlands Institute for Health Services Research.

Copyright * 2011 by Lippincott Williams \& Wilkins

Objectives: To develop a patient safety course for medical residents based on the views of medical residents and their supervisors.

Methods: In 2007, questionnaires were distributed to investigate residents' and supervisors' perspectives on the current patient safety performance and educational needs of residents. These perspectives were categorized according to the factors that influence daily practice as described in the London Protocol. Selection of course content and corresponding learning goals was made by an expert panel and based on the questionnaires' outcomes.

Results: One hundred sixteen (64\%) respondents filled out the questionnaire.

Residents rated health care as significantly safer than supervisors.

Close links were found between described risks and expressed educational needs. Both were found to be predominantly related to team factors, work environmental factors, and individual factors. The principal course themes that were selected are as follows: (1) principles of patient safety, (2) human factors, (3) effective teamwork, (4) contribution to safer care, and (5) medicolegal aspects of patient safety.

Conclusions: Residents are not fully aware of all potential risks of their work and of their own role in patient safety. This underlines the need for an explicit focus on patient safety issues during their training. A needs assessment among involved parties engages respondents in the process and can provide valuable input for developing patient safety education for residents.

Adverse events in health care have been the subject of numerous studies for the past 10 years. ${ }^{1-4}$ Specialists agree that the great number of these events could and should be significantly reduced by means of specific interventions. Patient safety education of health care workers is supposed to be one method that could contribute to patient safety improvement. ${ }^{5,6}$ For several reasons, it is expected that patient safety education for medical residents can lead to particularly valuable results.

First, residents provide much of the direct patient care.

Second, they are considered a fragile link in the care process, as research has revealed that a lack of work experience and high work pressure among residents increases risky situations. ${ }^{6,8}$ Besides, research showed that the patient safety knowledge of medical trainees across a broad range of training levels, degrees, and 
specialties was limited. ${ }^{9}$ A final argument for training residents in patient safety is that they are considered to be a group that can achieve long-lasting benefits, as these physicians are at the beginning of their career and they are the medical specialists of the future.

Since the extent of adverse events in health care became visible, many countries advocated a specific role for explicit patient safety education within the medical curriculum. ${ }^{10-12}$ Some articles were published that described the development of patient safety curricula for residents, ${ }^{6,13,14}$ but as far as we know, no studies integrated the perspectives of residents and educational supervisors. Educational development is a process consisting of 6 consecutive steps (Fig. 1), initiated by a perceived need. ${ }^{15}$ Assessment of learning needs (step 1) and analysis of desired behavior (step 2) are important at the start of any educational training and seem to be precursors of effective educational interventions. ${ }^{16} \mathrm{We}$ assessed residents' and supervisors' perspectives on patient safety performance and related educational needs (steps 1 and 2) and incorporated these into the definition of learning goals (step 3 ) and the selection of subjects for teaching patient safety (step 4).

\section{METHOD}

\section{Setting}

We developed a patient safety course for residents of a large Dutch regional teaching hospital where specialized care is being delivered. This hospital offers 21 different specialty training programs, and every year, approximately 85 graduate medical trainees fulfill (parts of) their residencies here. Simultaneously, approximately 50 medical graduates who are not (yet) in training to become a medical specialist contribute to the patients' care.

In our study, this latter group also was approached at assessing the residents' perspective. Therefore, "residents" in this paper refers to both groups of medical graduates.

Data Collection "Needs Assessment"

A 2-page questionnaire that contained closed questions and open-ended questions was developed by an expert panel of 3 physicians, 1 psychologist, 1 sociologist, and 1 health scientist with many years of experience in the field of patient safety research and medical education. We also consulted several patient associations. For this paper, we elaborate on the answers to 3 questions that provided the most interesting information. These questions are presented in Table 1. Before we distributed this questionnaire to all respondents, we pilot-tested it with representatives. From May 2007 until December 2007, we sent encoded paper versions of the questionnaire to the residents $(n=136)$ and to the supervisors participating in the hospital's committee of residency program directors $(n=46)$. In total, 182 questionnaires were sent. After 10 and 24 days, we sent reminders to lingering respondents.

\section{[FIGURE 1]}

\section{Analysis "Needs Assessment"}

We processed data in SPSS and used a probability of $\mathrm{P} \leq 0.05$ (2 tailed) to establish statistical significance. We assessed comparability of residents' characteristics for nominal measures by the $\mathrm{W}^{2}$ test and for scale measures by the independent samples $T$ test. To assess which unsafe aspects (question 2) were mentioned most, 1 author (J.D.J.) classified the answers according to the factors that were described in the London Protocol. ${ }^{17}$ This protocol distinguishes the following 7 factors that could influence daily practice: (1) patient factors, (2) task and technology factors, (3) individual (staff) factors, (4) team factors, (5) work environmental factors, (6) organizational and management factors, and (7) institutional context factors. If necessary, answers containing multiple aspects were classified according to multiple factors. For analyzing the first and second questions, we used the Mann-Whitney test because we wanted to compare 2 independent samples (residents versus the supervisors) on an ordinal scale. For analyzing question 1, we compared these groups according to the given answers. For the second question, we compared the number of aspects mentioned per group. For analyzing question 3, we used an inductive and iterative process, as our aim was to explore residents' and supervisors' perspectives with an open view, allowing the data to reveal patient safety educational themes. ${ }^{18}$ The answers were encoded by 1 author (J.D.J.) who was trained in qualitative research principles and had multiple years experience in conducting qualitative research. Any uncertainties about the classification of answers were discussed between the authors until consensus was reached. 


\section{[TABLE 1]}

\section{[TABLE 2]}

\section{Selection of Course Content}

The patient safety education expert panel reviewed the results of the needs assessment questionnaire to decide which patient safety themes should be included and which corresponding learning goals needed to be reached.

\section{RESULTS}

\section{Response}

In total, 116 respondents (64\%) completed the questionnaire: 91 residents $(67 \%)$ and 25 supervisors (54\%). Analysis showed that the responding residents formed a representative sample of the entire hospital's resident population. Table 2 shows background information on the respondents.

\section{Perceived Patient Safety Performance}

Analysis of the first question revealed that residents considered patient care in the hospital to be significantly safer $(\mathrm{P}=0.040)$ than supervisors (Fig. 2).

\section{Unsafe Aspects}

Question 2 was answered by 80 residents (88\%), revealing 119 aspects (mean, 1.5), and by 23 supervisors (92\%), uncovering 54 aspects (mean, 2.3) $(\mathrm{P}<0.001)$. One resident and 2 supervisors gave indecipherable answers that could not be analyzed. Nine residents did not answer the question.

Residents mostly mentioned work environmental factors, such as "high work pressure" and "too few health care workers per shift available." This was followed closely by team factors, for example, "poor writing in charts" and "relevant information gets lost at patient handovers." Individual (staff) factors also were frequently found in residents' answers, for instance, "inexperienced care deliverers" and "lack of knowledge about syndromes that are not directly related to own discipline.' In the supervisors' answers, these 3 factors also were frequently found. Many supervisors also mentioned task and technology factors, for example, "lack of unambiguous treatment plans"' and "bad computer system within the hospital." Table 3 shows the categorization of the unsafe aspects mentioned by residents and supervisors.

\section{[FIGURE 2]}

\section{[TABLE 3]}

\section{Patient Safety Learning Needs}

Sixty-three residents (69\%) and 23 supervisors (92\%) identified learning needs. Answers were closely related to the unsafe aspects that were mentioned at question 2 , although differences were found both between residents and supervisors and within these groups.

Fifty-seven $(90 \%)$ of the residents' answers showed that they prefer to learn skills for solving problems they experience in their daily work, for example, "what may I tell a patient after an incident has occurred," "how to improve the information transfer between health care workers," and "what and how can we learn from mistakes." Answers given by the supervisors more often focused on residents' fallibility and on being in a process of learning, such as "residents should become more aware of their own constraints and learn how to handle these properly," "more attention to the relevance of working with protocols," and " underline the need to be willing to receive feedback." Table 4 displays a selection of the educational needs that were expressed.

\section{Course Content}

The patient safety education expert panel reviewed the learning needs identified by the respondents to decide which patient safety themes should be included and which corresponding educational objectives needed to be reached. We used a major part of the educational needs that resulted from the questionnaire for defining 5 course themes and corresponding educational objectives (Table 4). Theme 1 covers the principles of patient safety that stimulate awareness and provide a foundation for learning about the other themes. Theme 2 concentrates on the role of human factors in patient safety. Themes 3 and 4 both predominantly focus on what one can do to improve the safety of patients. Theme 5 mainly focuses on the 
Jansma, J.D., Wagner, C., Bijnen, A.B. A patient safety curriculum for medical residents based on the perspectives of residents and supervisors. Journal of Patient Safety: 2011, 7(2), 99-105

required response after incidents have occurred. The major difference between themes 1 and 2 and the and 4 is that the first 2 themes are predominantly focusing on theories and research about patient safety (i.e., patient safety definitions, process of incident occurrence, scope of patient safety problems), whereas themes 3 and 4 focus more on residents' daily practice (i.e., offering methods to improve patient safety, exercising with specific patient safety improvement tools). The 3 patient safety factors that were mentioned most frequently in the answers to the needs assessment (Table 3 ) were explicitly integrated into the course. Teamwork factors are primarily incorporated into theme 3 ; work environmental factors are mainly covered by themes 4 and 5 , and individual (staff) factors are dealt within themes $2,3,4$, and 5 .

\section{[TABLE 4]}

\section{DISCUSSION}

Patient safety education has gained more attention within medical education. This paper described the developmental process and content of a multispecialty patient safety course for residents. To that purpose, we sought the opinion of both residents and their supervisors on patient safety performance and patient safety educational needs. The complemental views of both parties were very useful for the development of a patient safety course for residents. The principal course themes that we selected from these outcomes are as follows: (1) principles of patient safety, (2) human factors, (3) effective teamwork, (4) contribution to safer care, and (5) medicolegal aspects of patient safety.

Residents considered patient care to be significantly safer than their experienced supervisors. This is interesting to note, as residents are considered a fragile link in the care process. ${ }^{6,8}$ Besides, residents mainly focused on work environmental factors that interfere with patient safety, whereas supervisors more often addressed residents' own fallibility and the risks connected to the process of learning. This strongly suggests that residents are not fully aware of their own role in patient safety, and it stresses the need for more attention to patient safety as a specific component of their training.

The course themes we selected largely correspond with existing literature on what is important for patient safety education.

${ }_{6,13}$ It is expected that most of the patient safety factors of the London Protocol can be (partly) influenced by making residents more conscious of patient safety issues. For example, increasing residents' safety knowledge and skills can make them more competent at signaling risky situations in an early stage and handling them properly (individual [staff] factors).

Improvement of written and verbal communication within the team (team factors), being able to find and follow protocols (task and technology factors), and discussing patterns of shifts and workload (work environmental factors) could contribute to patient safety improvement. Educating residents will not directly influence external factors (patient factors, organizational and management factors, and institutional context factors), but education could enable residents to signal these risky factors more readily.

\section{Didactical Design}

A subsequent step in educational development is the didactical design (step 5, Fig. 1), that is, the selection of educational methods to achieve the learning goals. Thereby, didactical principles should be kept in mind. Four major didactical principles include the following: (1) Education needs to be closely related to clinical practice, and wherever possible, it needs to be incorporated in the attendants' daily practice because what people learn in 1 context will not necessarily apply in another setting. ${ }^{19}$ (2) Adult learning theorists have recognized that an interactive environment with multispecialty small groups stimulates the learning process by enabling optimal learning from peers. ${ }^{5}$ (3) Education in multiple sessions often is more effective than education consisting of a single session. Coyle et $\mathrm{al}^{20}$ demonstrated that patient safety education had more impact if residents had attended multiple educational sessions.

(4) Assessment stimulates learning. ${ }^{21}$ Workshops, practice assignments, mentoring, and assessments are among the educational methods in line with these didactical principles.

\section{Workshops}

The needs assessment made it clear that poor communication between health care workers is considered an unsafe aspect of health care and that a practical training would be helpful.

We addressed this in workshops in small groups, focusing on handovers between health care workers. The usefulness of structured transfer of information, for example, according to the Situation Background Assessment Recommendation technique, ${ }^{22}$ was explained and practiced. This made residents aware of risky 
aspects and offered strategies to improve the information transfer in daily practice. Part of the workshbe consisted of role playing, an educational method that had been considered valuable by patient safety course attendants elsewhere. ${ }^{23}$ This workshop addressed elements of themes 2, 3, and 4.

\section{Practice Assignments}

The supervisors would have liked residents to become more aware of the occurrence of risky situations in their daily practice and to learn how to handle these situations properly. To address these needs, we gave practice assignments that stimulated the attendants' focus on risky situations in their daily work. We asked residents to make a brief description of 10 incidents they noticed between the first and the second course meeting. To that purpose, we provided pocket-size reporting cards that attendants could put into their white coats. To make attendants more aware of the underlying causes, we also asked them to perform a root cause analysis on 5 of these incidents. ${ }^{24}$ The attendants' experiences with carrying out these assignments were the topic of a group discussion during the second course meeting, creating an interactive learning environment. These assignments were in line with educational objectives of themes 1, 2, and 4.

\section{Mentoring}

We recruited experienced medical professionals (i.e., specialists and nurses) working at the same hospital for voluntary participation as a mentor, to encourage the learning process and stimulate fulfillment of the practice assignments. All mentors received specific instructions, including an explanation of the main patient safety principles and the course content. They were available to give residents advice, counseling, and developmental opportunities in relation to patient safety. An open culture is considered essential toward improving the safety of patients. ${ }^{25}$ To avoid potential barriers to such openness as a consequence of (hierarchical) dependence, ${ }^{26}$ we brought together mentors and residents from different disciplines.

\section{Assessments and Incentives}

To stimulate learning, we included a knowledge assessment and announced this at the beginning of the course. Apart from a summative role for this assessment in evaluating the results of this training, we discussed the answers with the residents afterward, to provide an additional formative learning moment. As an additional motivation, we provided official certificates and desirable incentives to residents with good assessment results.

For example, specially made patient safety pins, which attendants can wear on their white coats, could be obtained. In addition, we assigned honorable mentions on certificates for special efforts, such as for the most active attendant and for the highest score at the knowledge assessment.

The selection of educational methods also depends on the available opportunities within the organization, that is, budget, time, and location. In line with the feasibility in our current setting, the patient safety course consisted of 1 plenary day (18-24 attendants), followed by 2 half days in smaller groups (6-8 attendants) with a 4- to 6-week interval. At this course, residents from various disciplines were learning together. The educational methods that we chose for our setting included workshops, practice assignments, mentoring, and assessments.

\section{Limitations}

It is important to keep in mind that this study largely depended on subjective measures. Focused was on residents' and supervisors' views, but we think that other parties involved, such as nurses, also could provide a valuable input. Residents may lack insight or awareness of some of the significant content that is relevant to patient safety education; therefore, we also assessed the views of their (more experienced) supervisors. Moreover, the educational content and corresponding learning goals were ultimately chosen by a panel of experts in the field of patient safety, to prevent important patient safety topics from being missed, such as causes of cognitive errors. This study included residents and supervisors of 1 hospital; therefore, generalizations must be made with some caution. However, the respondents can be considered a dynamic group with experience in diverse settings and national and international networks, and it is nevertheless reasonable to assume that this study is relevant for developing patient safety curricula elsewhere.

\section{Further Research}

A further important step in educational development that was not addressed in this paper is the evaluation of the education (step 6, Fig. 1). The effectiveness of the curriculum has to be evaluated, as a guide for further improvement mainly. ${ }^{27}$ Preferably, such an evaluation should be performed with control groups. ${ }^{27}$ Although the preferred outcome of an educational program is a change in behavior, the potential precursors of such a change, like changes in attitudes and intentions, can often be measured more easily and are 
Jansma, J.D., Wagner, C., Bijnen, A.B. A patient safety curriculum for medical residents based on the perspectives of residents and supervisors. Journal of Patient Safety: 2011, 7(2), 99-105

predictors for an actual behavioral change. ${ }^{28}$ Such a measurement has been performed and will be thive subject of another publication. ${ }^{29}$

\section{CONCLUSIONS}

Residents are not fully aware of all potential risks of their work and of their own role in patient safety. This underlines the need for an explicit focus on patient safety issues during their training. A needs assessment among involved parties can provide valuable input for developing patient safety education for residents and engages respondents in the development process.

Education for residents is only a small step toward a safer environment for patients. To improve safety, it is recommended to educate not only residents but also all health care workers who contribute to the care process. Moreover, also, organizational factors, such as flaws in the computer system or an inadequate arrangement of the medicine cupboard, should be assessed as well and improved if necessary. However, to become aware of the risky factors and to become skilled at handling them properly, education is an essential facilitator.

\section{REFERENCES}

1. Brennan TA, Leape LL, Laird NM, et al. Incidence of adverse events and negligence in hospitalized patients. Results of the Harvard Medical Practice Study I. N Engl J Med. 1991;324:370Y376.

2. Kohn LT, Corrigan JM, Donaldson MS. To Err is Human, Building a Safer Healthcare System. 2000.

3. Vincent C, Neale G, Woloshynowych M. Adverse events in British hospitals: preliminary retrospective record review. Br Med J.

2001;322:517Y519.

4. Zegers $M$, de Bruijne MC, Wagner $C$, et al. Adverse events and potentially preventable deaths in Dutch hospitals. Results of a retrospective patient record review study. Qual Saf Health Care. 2009.

5. Henriksen K, Dayton E. Issues in the design of training for quality and safety. Qual Saf Health Care. 2006;15(suppl 1):i17Yi24.

6. Heffner JE, Ellis R. Training resident physicians toward improved patient safety. J S C Med Assoc. 2004;100:168Y171.

7. Volpp KG, Grande D. Residents' suggestions for reducing errors in teaching hospitals. N Engl J Med. 2003;348:851Y855.

8. Singh $\mathrm{H}$, Thomas EJ, Petersen LA, et al. Medical errors involving trainees: a study of closed malpractice claims from 5 insurers.

Arch Intern Med. 2007;167:2030Y2036.

9. Kerfoot BP, Conlin PR, Travison T, et al. Patient safety knowledge and its determinants in medical trainees. J Gen Intern Med.

2007;22:1150Y1154.

10. COGME, NACNEP. Collaborative education to ensure patient safety.

2000. Council on Graduate Medical Education and the National Advisory Council on Nurse Education and Practice. Ref Type: Report 11. Greiner AC. Health Professions Education: A Bridge to Quality.

Washington, DC: National Academies Press; 2003.

12. NVZ, OMS, LEV\&V, VVN, NFUMC. Veiligheidsprogramma Voorkom schade, werk veilig in de Nederlandse ziekenhuizen. 2007.

NVZ vereniging van ziekenhuizen, Orde van Medisch Specialisten, Landelijk Expertisecentrum Verpleging \& Verzorging, Verpleegkundigen \& Verzorgenden Nederland en Nederlandse Federatie van Universitair Medische Centra. Ref Type: Report 13. Varkey P, Karlapudi S, Rose S, et al. A patient safety curriculum for graduate medical education: results from a needs assessment of educators and patient safety experts. Am J Med Qual. 2009;24:214Y221.

14. Singh R, Naughton B, Taylor JS, et al. A comprehensive collaborative patient safety residency curriculum to address the ACGME core competencies. Med Educ. 2005;39:1195Y1204.

15. Kern DE, Thomas PA, Howard DM, et al. Curriculum development for medical education: a six-step approach. The John Hopkins University Press; 1998.

16. Davis DA, Thomson MA, Oxman AD, et al. Changing physician performance. A systematic review of the effect of continuing medical education strategies. JAMA. 1995;274:700Y705.

17. Taylor-Adams S, Vincent C. Systems analysis of clinical incidents: The London protocol. 2006. Clinical Safety Research Unit. Ref Type: Report 18. Strauss AL, Corin J. Basics of Qualitative Research: Grounded Theory Procedures and Techniques. Newbury Park, CA: Sage Publications; 1990.

19. Perkins DN, Salomon G. International Encyclopedia of Education, Transfer of Learning. 2nd ed. Oxford, UK: Pergamon Press; 2002. 
Jansma, J.D., Wagner, C., Bijnen, A.B. A patient safety curriculum for medical residents based on the perspectives of residents and supervisors. Journal of Patient Safety: 2011, 7(2), 99-105

20. Coyle YM, Mercer SQ, Murphy-Cullen CL, et al. Effectiveness of a graduate medical education for improving medical event reporting attitude and behavior. Qual Saf Health Care. 2005;14: 383 Y388.

21. Epstein RM. Assessment in medical education. N Engl J Med. 2007; 356:387Y396.

22. Haig KM, Sutton S, Whittington J. SBAR: a shared mental model for improving communication between clinicians. Jt Comm J Qual Patient Saf. 2006;32:167Y175.

23. Patey $\mathrm{R}$, Flin $\mathrm{R}$, Cuthbertson $\mathrm{BH}$, et al. Patient safety: helping medical students understand error in healthcare. Qual Saf Health Care. 2007;16:256Y259.

24. Van der Schaaf TJ, Habraken MMP. PRISMA methode medische versie [PRISMA method medical version]. 2005. Ref Type: Report 25. Institute of Medicine. Crossing the Quality Chasm: A New Health System for the 21st Century. Washington, DC, National Academy Press; 2001. Ref Type: Report 26. Kobayashi H, Pian-Smith M, Sato M, et al. A cross-cultural survey of residents' perceived barriers in questioning/challenging authority.

Qual Saf Health Care. 2006;15:277Y283.

27. Craig P, Dieppe P, Macintyre S, et al. Developing and evaluating complex interventions: the new Medical Research Council guidance.

BMJ. 2008;337:a1655.

28. Ajzen I, Fishbein M. The influence of attitudes on behavior. In: Albarracín D, Johnson BT, Zanna MP, eds. The Handbook of Attitudes.

Mahwah, Mahwah, NJ: Erlbaum; 2005. 173Y221.

29. Jansma JD, Leistikow IP, Kalkman CJ, et al. Do specialty registrars change their attitudes, intentions and behaviour towards reporting incidents following a patient safety course? BMC Health Services Research. In press. 


\section{TABLES AND FIGURES}

Figure 1

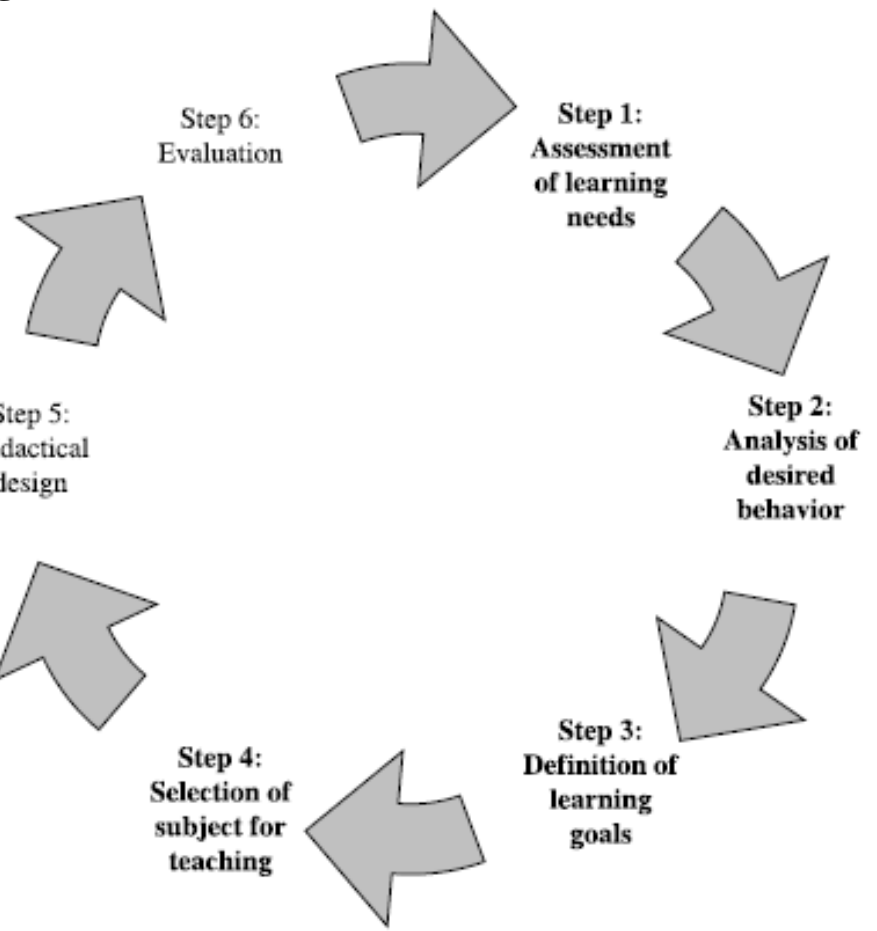

FIGURE 1. Steps in educational development (steps $1-4$ of the educational development circle are addressed in this manuscript).

Table 1

TABLE 1. Part of Needs Assessment Questionnaire

\begin{tabular}{ll}
\hline Questions & \multicolumn{1}{c}{ Answer Options } \\
\hline 1. How would you describe the safety of patients in your hospital? & $\begin{array}{l}\text { Likert scale: 1, extremely unsafe; 2, unsafe; 3, } \\
\text { neither unsafe nor safe; 4, safe; 5, extremely safe } \\
\text { Open question }\end{array}$ \\
2. Which aspects contribute to unsafe situations within your work? & $\begin{array}{l}\text { Open question } \\
\text { 3. What do you hope residents will learn from a patient safety course? }\end{array}$ \\
\hline
\end{tabular}

\section{Table 2}

TABLE 2. Characteristics of Respondents

\begin{tabular}{lcc}
\hline Characteristics & $\begin{array}{c}\text { Residents } \\
(\mathbf{n = 9 1 )}\end{array}$ & $\begin{array}{c}\text { Supervisors } \\
(\mathbf{n}=\mathbf{2 5})\end{array}$ \\
\hline $\begin{array}{l}\text { Age, yr } \\
\text { Range } \\
\text { Median age }\end{array}$ & $25-53$ & $40-60$ \\
Sex, n (\%) & 31 & 48 \\
$\quad$ Male & & \\
$\quad$ Female & $34(37)$ & $23(92)$ \\
Discipline, n (\%) & $57(63)$ & $2(8)$ \\
$\quad$ Surgical & & $9(36)$ \\
$\quad$ Nonsurgical & $26(29)$ & $16(64)$ \\
Residency training, n (\%) & $65(71)$ & \\
$\quad$ Yes & & \\
No & $60(66)$ & \\
\hline
\end{tabular}


Jansma, J.D., Wagner, C., Bijnen, A.B. A patient safety curriculum for medical residents based on the perspectives of residents and supervisors. Journal of Patient Safety: 2011, 7(2), 99-105

Figure 2
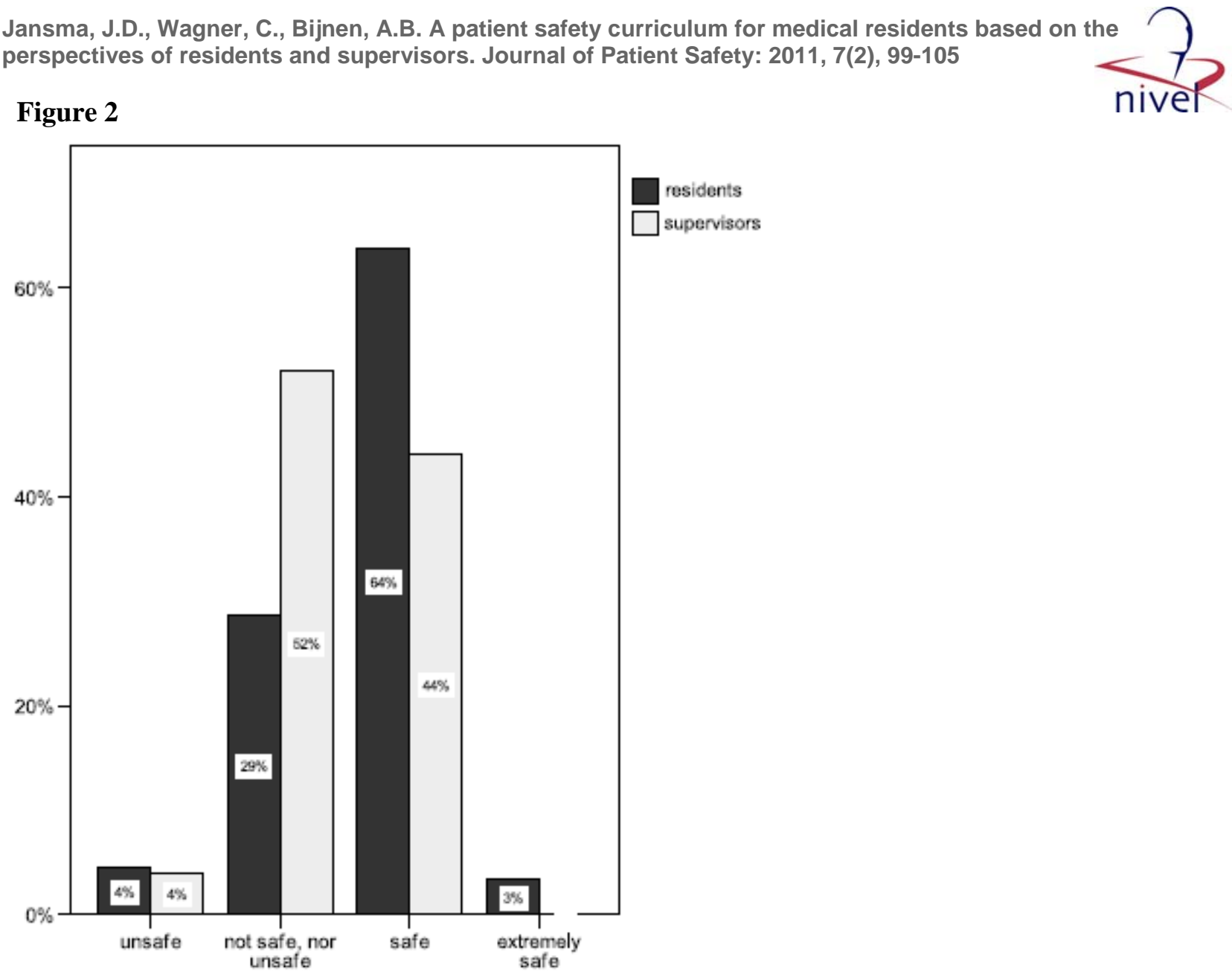

FIGURE 2. Perception of in-hospital patient safety (none of the respondents declared to perceive in-hospital patient safety

extremely unsafe). Residents $(n=91)$ and supervisors $(n=25)$.

\section{Table 3}

TABLE 3. Categorization of Unsafe Aspects Mentioned by Residents $(n=119)$ and Supervisors $(n=54)$

\begin{tabular}{llcc}
\hline Patient Safety Factors & \multicolumn{1}{c}{ Unsafe Aspects Mentioned } & Residents n (\%) & Supervisors n (\%) \\
\hline Patient factors & Linguistic barrier & $4(3)$ & $1(2)$ \\
Task and technology factors & Bad task design & $15(13)$ & $7(13)$ \\
& $\begin{array}{l}\text { Unavailability and inadequate use of protocols } \\
\text { Unavailability and inaccuracy of test results }\end{array}$ & $16(13)$ & $10(19)$ \\
Individual (staff) factors & $\begin{array}{l}\text { Lack of knowledge and skills } \\
\text { Incompetence }\end{array}$ & $39(33)$ & $17(32)$ \\
Team factors & $\begin{array}{l}\text { Poor verbal communication } \\
\text { Poor written communication }\end{array}$ & $42(35)$ & $15(28)$ \\
Work environmental factors & $\begin{array}{l}\text { High workload } \\
\text { Bad design, lack of availability and inadequate } \\
\text { maintenance of equipment }\end{array}$ & $3(3)$ & 0 \\
Organizational and management factors & Poor organizational structure & $3(6)$ \\
Institutional context factors & Incorrect use of health services & & $1(2)$ \\
\hline
\end{tabular}


Jansma, J.D., Wagner, C., Bijnen, A.B. A patient safety curriculum for medical residents based on the perspectives of residents and supervisors. Journal of Patient Safety: 2011, 7(2), 99-105

Table 4

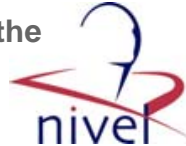

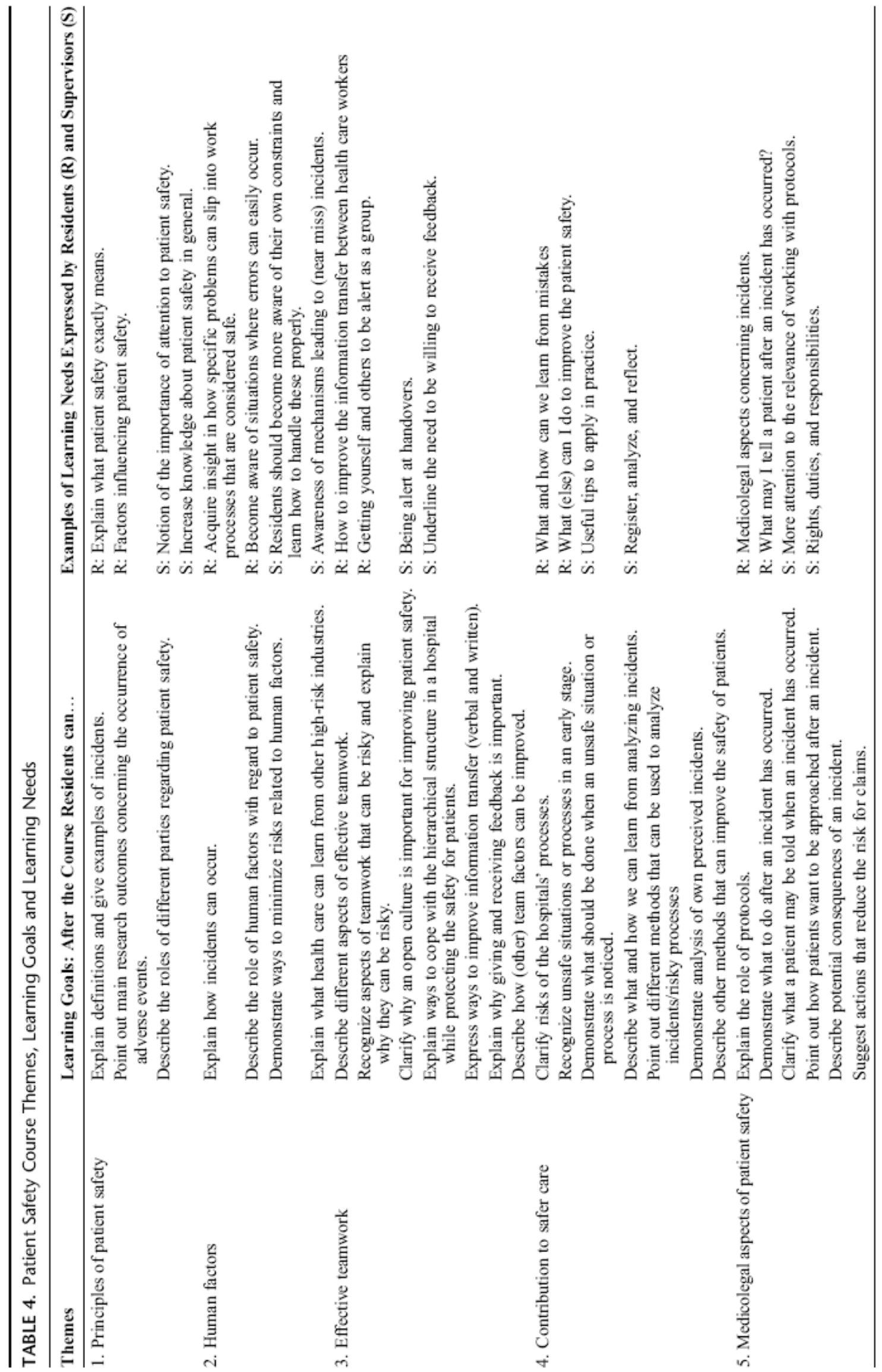

